\title{
Locomotion study of a single actuated, modular swimming robot
}

\author{
M. Fremerey, L. Fischheiter, J. Mämpel \& H. Witte \\ Department of Biomechatronics, Faculty of Mechanical Engineering, \\ Ilmenau University of Technology, Germany
}

\begin{abstract}
This paper introduces a concept for a biologically inspired modular swimming robot, which is able to perform different swimming styles of steady swimming species. Therefore the robot has two kinds of modules: 1. a basic structure, which creates the required torque and determines the swimming direction of the robot; 2. effector modules that generate the robot's propulsion in interaction with the surrounding liquid. Due to the specific task, the effector modules are exchangeable.

This approach enables the gain of technical and biological knowledge: The results of research tend to a design that is suited to fulfill tasks in particular situations when common marine propeller driven robots are not able to work (e.g. an environment with many plants). In addition, the permutation of bio-analogous features in a robot improves understanding of the biological antetype.

The current design of the effector modules is inspired by undulant swimming species, mainly sea snakes and eels. Biological research and mathematical modeling yielded to a three segmented tail structure. The coupling of the segments to each other uses spring elements.
\end{abstract}

Keywords: biologically inspired robots, snake-like robot, aquatic robots, modular robots, steady swimming fishes.

\section{Introduction}

Research in the locomotion of steady swimming aquatic animals (Sfakiotakis et al. [10]) offers numerous inspirations for technical solutions. 
The generation of propulsion without the hitherto common propeller seems to be necessary for extending the range of possible applications. Robots are able to operate in sensitive ecological environments, e.g. with many plants, algae or other organisms. In addition, such drives could reduce the noise emission that current technical systems induce to water.

Biologically inspired robotics provides a suitable answer for getting access to principles that underlie these features. Transfer from nature to robotics is done by reducing the observed biological ability to its basic principle and by using common engineering strategies in modeling and design.

\section{Biological background}

Fish propulsion of steady swimming fishes is divisible into four main classes: 1 . anguilliform, 2. carangiform, 3. thunniform and 4. ostraciiform (Sfakiotakis et al. [10]). These swimming modes differ as well in relative body length acting against the water as in amplitude of body movement (compare to figure 2).

For the current design a variant of the anguilliform propulsion is realized: the slow anguilliform swimming style.

Based upon the research of Laulder and Tytell [7], eels show a difference in body length acting against the water subjected to swimming velocity. While swimming with velocities greater than one body length per second, they produce a rhythmic lateral elongation with raising amplitude from head to tail using at least two thirds of their body. By contrast while swimming with velocities below one body length per second, eels elongate only the last third of body. The strong curved S-shape shown during normal swimming is not formed.

These results provide the transfer to a modular robot featuring a single drive for creating propulsion.

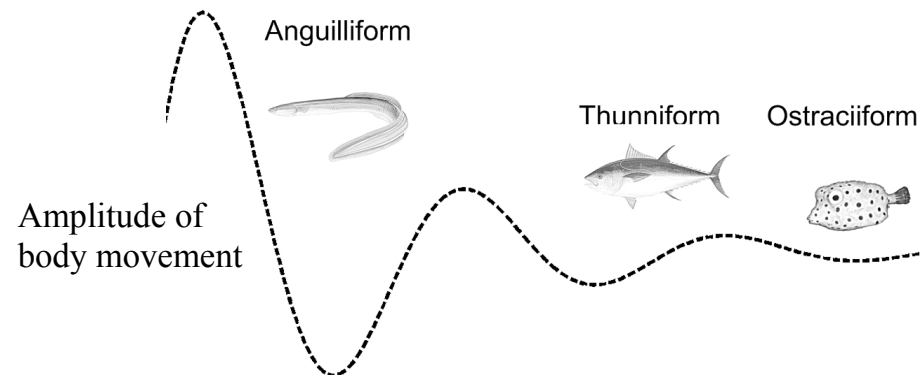

Figure 1: From anguilliform to ostraciiform the swimmers' body length acts against the water and the amplitude of body movement lowers. In reverse the flap frequency rises (figure source Sfakiotakis et al. [10]). 


\section{Model of the modular swimming robot}

In order to investigate several characteristics of different kinds of steady swimmers with less effort, a modular robot is introduced, featuring two different types of modules:

- The basic structure, which creates the required torque and determines the direction independent from the specific swimming mode

- Effector modules, which transmit the torque to the surrounding water and enable the specific swimming mode. They are exchangeable.

Defined attaching and detaching of mass, energy and information flux at the borders of basic structure and effector modules enables a change in the overall function of the robot. The system becomes versatilely deployable (Mämpel et al. [8], Zhang et al. [11]).

Subsidiary this proceeding benefits a robot design which reduces the amount of actuators creating propulsion to the minimum number of one. This approach differs from the current state of the art, where a drive in each module is realized (Crespi et al. [2], Hirose [12], DFKI [13]).

At the moment, the design of effector modules implements the principles of slow anguilliform swimmers, taking the inspiration mainly from sea snakes and eels.

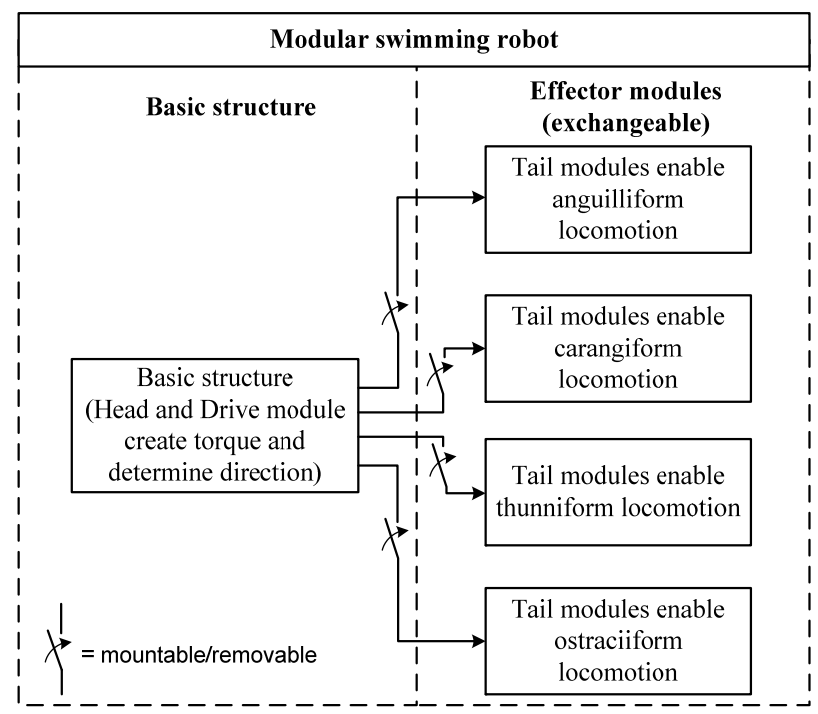

Figure 2: $\quad$ Types of modules of the modular swimming robot: it consists of a basic structure and effector modules. Thereby, effector modules are exchangeable for realizing different setups for research with a single basic structure. 
By creating the locomotion via mechanically coupled effector modules, the robot additionally enables the investigation of the interaction of different kinds of compliance subjected to the propulsion.

These different kinds of compliance are mainly the compliance of the fluidic environment (extrinsic compliance) and the compliance of the robot (intrinsic compliance) in connection with its inertia.

\subsection{Model setup}

For investigating the propulsive force of a slow anguilliform oscillating tail, a mathematical model is introduced. Reducing the continuous compliance of the antetype (sea snake) to discrete compliant joints allows usage of the theories of rigid body mechanics.

Therewith the model consists of several rigid segments coupled by springs. Due to the desired modularity, the modules are homogeneous in form and size. The connection between each module occurs via leg springs. The bedding of the model forms a one side fix-bedding. A singular force emulates the resistance of the surrounding water. It affects the center of mass (successive COM) of each module. Figure 3 introduces all necessary data.

The differential equation of the tail is describable by

$$
J * \ddot{\varphi}=\sum_{i=1}^{n} M_{i}
$$

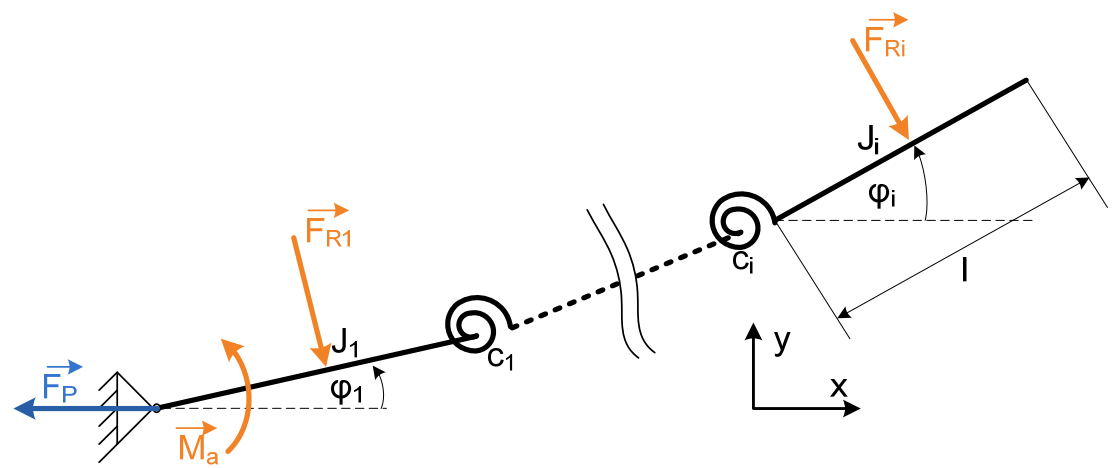

Figure 3: Multi-body model of the tail of the aquatic robot, with $c_{1} \ldots c_{i}=$ spring coefficients, $\varphi_{1} \ldots \varphi_{i}$ elongation of the modules subjected to a parallel shifted $\mathrm{x}$-axis, $\mathrm{F}_{\mathrm{R} 1} \ldots \mathrm{F}_{\mathrm{Ri}}$ resistance force of the surrounding water, $\mathrm{F}_{\mathrm{p}}$ resulting propulsive force in $\mathrm{x}$-direction and applied torque $\mathrm{M}_{\mathrm{a}}$. 
With the assumption of a static fluid the resistance force of the surrounding water results to:

$$
\begin{array}{r}
\boldsymbol{F}_{\boldsymbol{R}}=\frac{\mathbf{1}}{\mathbf{2}} * \boldsymbol{\rho} * \boldsymbol{c}_{\boldsymbol{w}} * A * \boldsymbol{v}^{2} \\
M_{\boldsymbol{R}}=\frac{\boldsymbol{l}^{3}}{\mathbf{1 6}} * \rho * c_{w} * A * \dot{\varphi}_{i}^{2}
\end{array}
$$

Due to the effective direction of the resistance force opposite to the direction of movement, the signum function is introduced.

$$
M_{R}=-\operatorname{sign}\left(\dot{\varphi}_{i}\right) * D * \dot{\varphi}_{i}^{2}
$$

$\rho$ represents the density, $c_{w}$ the drag coefficient, A the projected area of the body which resists the water and $v$ the velocity of the elongated body. The number of segment labels i. Therewith the modeling of damping in the joints is negligible; the tail of an anguilliform swimmer is expressed by:

$$
\begin{gathered}
J_{1} * \ddot{\varphi}_{1}=M_{a}-c_{1}\left(\varphi_{1}-\varphi_{2}\right)-\operatorname{sign}\left(\dot{\varphi}_{1}\right) * D * \dot{\varphi}_{1}^{2} \\
J_{2} * \ddot{\varphi}_{2}=-c_{1}\left(\varphi_{2}-\varphi_{1}\right)-c_{2}\left(\varphi_{2}-\varphi_{3}\right)-\operatorname{sign}\left(\dot{\varphi}_{2}\right) * D * \dot{\varphi}_{2}^{2} \\
J_{i} * \ddot{\varphi}_{i}=-c_{i-1}\left(\varphi_{i}-\varphi_{i-1}\right)-c_{i}\left(\varphi_{i}-\varphi_{i+1}\right)-\operatorname{sign}\left(\dot{\varphi}_{i}\right) * D * \dot{\varphi}_{i}^{2}
\end{gathered}
$$

Simulation environment and procedure

The software tool ADAMS was used for analyzing the multi body system. Required torque is determined by forcing a motion in form of a sinusoidal wave to the first module of the tail. Applying this torque in reverse to the whole model of the tail enables the representation of the resulting propulsion.

In addition a systematic parameter variation is executed in order to gain constructional data concerning the expected spring stiffness. Impressing the sinusoidal motion to the first tail module, the spring stiffness of each spring involved is altered. Tracking the resulting difference in swing characteristics allows the selection of suitable values for spring stiffness.

During simulation, quantity and length of tail modules are changed.

\subsection{Results of the systematic research on motion parameters}

Table 1 summarizes the configuration of the tail achieving best swinging characteristics.

Table 1: Configuration enabling best swinging characteristics.

\begin{tabular}{c|c|c|c|c}
\hline $\begin{array}{c}\text { number of } \\
\text { modules }\end{array}$ & $\begin{array}{c}\text { shape of a } \\
\text { module }\end{array}$ & $\begin{array}{c}\text { length of a } \\
\text { module in } \\
\mathrm{mm}\end{array}$ & $\begin{array}{c}\text { width of a } \\
\text { module } \\
\text { in mm }\end{array}$ & $\begin{array}{c}\text { number } \\
\text { of joints }\end{array}$ \\
\hline 3 & cylinder & 200 & 50 & 2 \\
\hline
\end{tabular}


The simulation shows three states concerning the behavior of the tail: 1. First state occurs simulating very soft springs. The whole tail oscillates with raising amplitude (hypercritical gain) and flips over at a certain point. As consequence the tail is damaged or even destroyed. 2. While choosing the spring stiffness too stiff, the tail modules act like a single rigid body during elongation: The desired compliance is nonexistent. 3. Assuming the spring stiffness between state 1 and 2 tends to a swinging behavior close to the biological antetype. The tail oscillates smoothly from left to right; the range of applicable spring stiffnesses is shown in figure 4.

Based upon the systematic research best swinging characteristics are achieved by reducing the spring stiffness from head to tail by a factor of about 10 regarding each joining.

Setting the spring stiffness around the optimum shown in figure 4, the driving torque averages $0.02 \mathrm{Nm}$ which means a net propulsion of $0.02 \mathrm{~N}$. Due to the positive sign it enables a forward motion of the robot.

\section{Design of the modular swimming robot}

\subsection{Design of the modules and chosen material}

The design process follows the common guide line for development of mechatronical systems (VDI 2206 [6]).

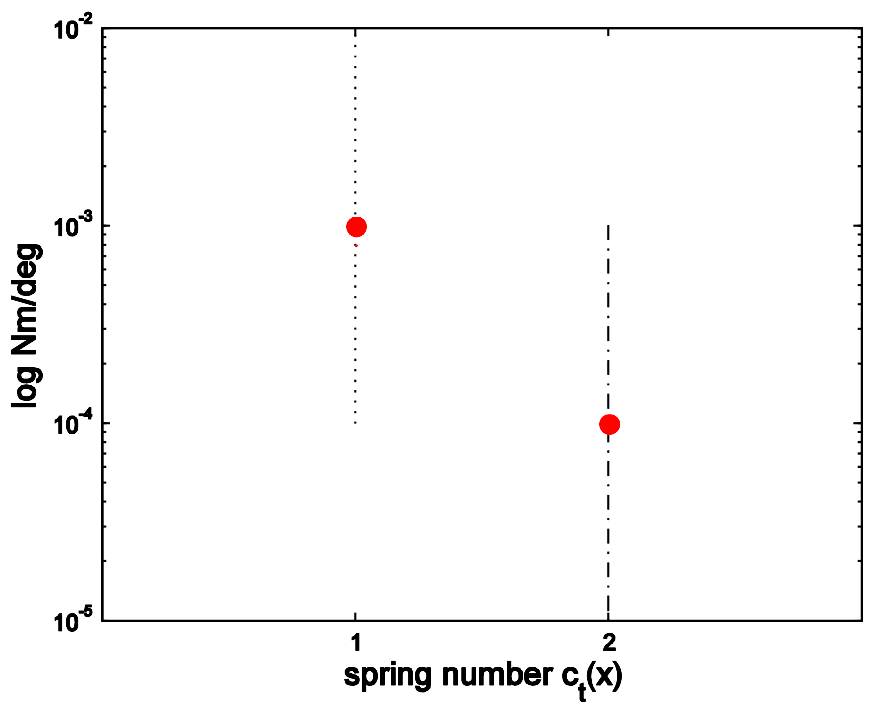

Figure 4: Simulated range of adequate spring stiffnesses of the three segmented tails. Red dots represent the simulated optimum for each leg spring where tail swings are the best (color online only). If the stiffness chosen is too soft, the modules buckle. Compliance is nonexistent if the stiffness chosen is too tough. 
All modules are made from acrylic glass due to its resistance against salt water and to aesthetic reasons. In addition, the clear-transparent acrylic glass enables the immediate detection of possibly appearing problems. According to the mathematical model, the modules are cylinders with $200 \mathrm{~mm}$ length and 50 $\mathrm{mm}$ width. An insertion plate inside each cylinder contains all required technical internals.

\subsection{Design of the basic structure}

The basic structure consists of two modules, due to the two main functions: Creating propulsion and changing the direction of the robot. The required torque provides an actuator-gear combination. In order to reduce the amount of electronic, a cam mechanism translates the continuous rotation of the drive into an alternating one. Therewith an elongation of the first effector module of about $+/-15$ degree is possible, with a maximum torque of $3 \mathrm{Nm}$. A change in direction

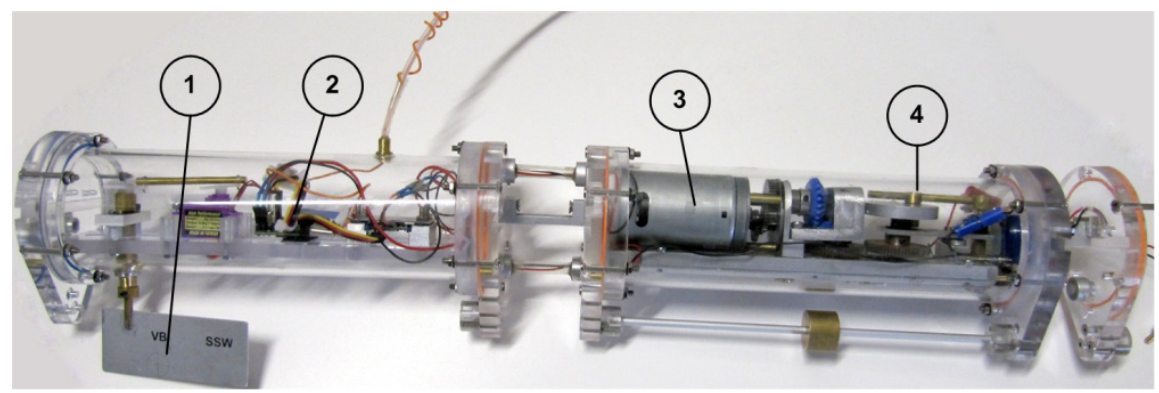

Figure 5: The two modules of the basic structure: $1=$ helm, $2=$ receiver, $3=$ motor + gear, $4=$ cam mechanism.

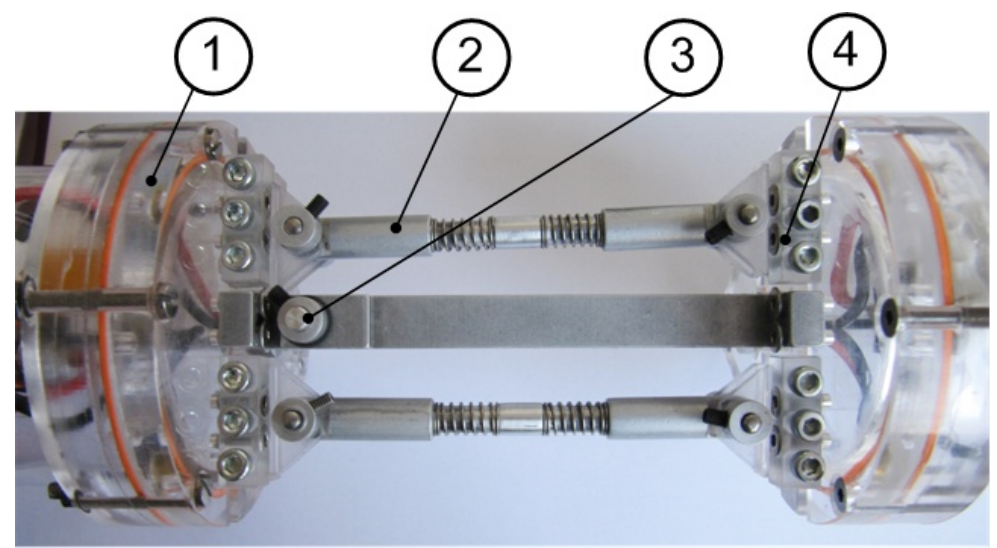

Figure 6: Connection between two tail modules: $1=$ tail module, $2=$ tuneable spring element, $3=$ rigid revolute joint. The spring elements are mounted to a slidable bedding (4). 


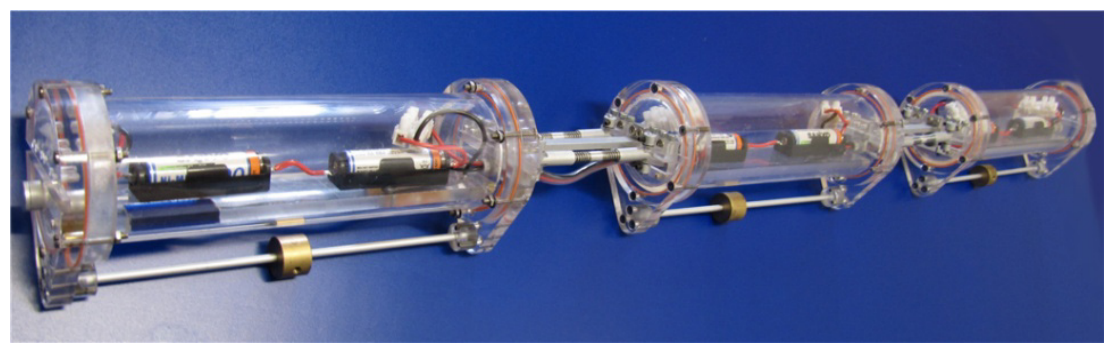

Figure 7: Design of the three-segmented tail, the overall length averages $\approx$ $800 \mathrm{~mm}$.

facilitates a thin metal sheet, fixed to a rotatable lever. To avoid water inleakage, the rotatable lever is bedded into a stern tube construction. Furthermore, the basic structure carries motor control, receiver and required energy.

\subsection{Design of the current effector modules}

According to the mathematical modeling, the "tail" consists of three modules. A revolute joint enables the rigid connection between the modules. The intrinsic compliance of each joint is realized by linear spring elements. These translational elasticities replace the rotational springs used during simulation to facilitate technical implementation. The spring elements feature an active spring calibration for tuning the intrinsic compliance: By sliding the bedding of the element to the left and right the lever ratio changes (compare to figure 6).

Figure 7 shows the three-segmented tail.

\subsection{Electronics and control}

At present, the robot is remote controlled by a user. For this purpose, a standard wireless communication is used. Voltage of drive and the position of the helm are controlled in open-loop, due to the hitherto not implemented sensory system. Staying at the surface of water, this kind of communication is adequate.

The signals are routed to the drive by a common cruise control. Lithium Metal-Hybrid batteries provides the required energy. Their weight additionally is used to balance the robot.

\section{Experiments}

To analyze the designed tail, a function test is introduced. Therefore tail module 1 is manually forced to oscillate with an amplitude of about $+/-15$ degree. Figure 8 summarizes the test results as a frame sequence.

By forcing module 1 to the right (1), module 2 and 3 follow the impressed motion in (2). The elongation of module 3 rises from (2) to (4). In frame (5) the sense of direction of the impressed torque alters: module 1 is forced to the left. Due to the inertia of the system, module 3 reaches the maximum of elongation in frame (5), too. From (5) to (7), module 2 and 3 obey the motion of module 1 again: they move to the left. 


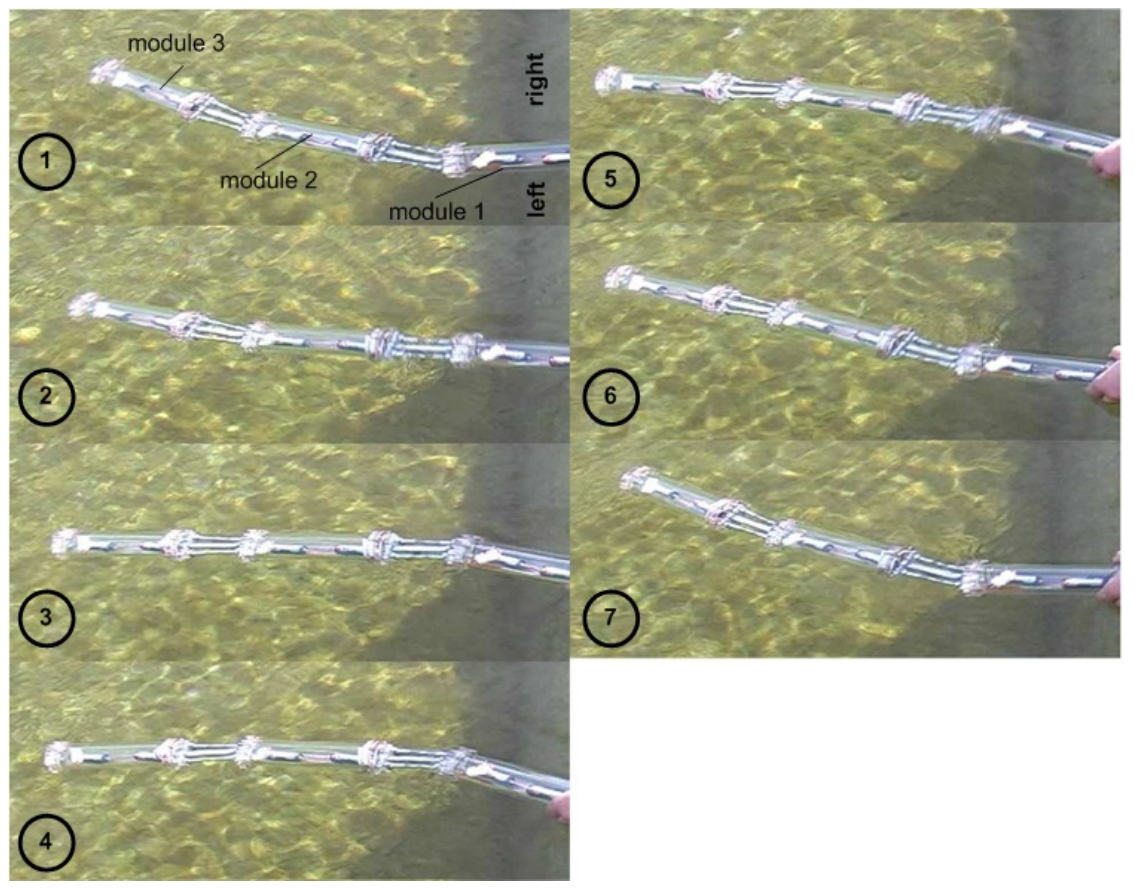

Figure 8: $\quad$ Motion capture of three manually deflected modules.

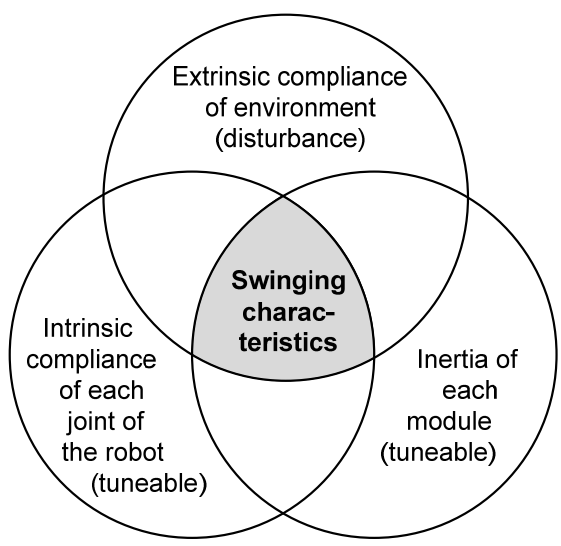

Figure 9: Interaction of extrinsic compliance, intrinsic compliance and module inertia concerning the swinging characteristics. Tuneable intrinsic compliance along with the tuneable inertia of each module encounters the disturbing extrinsic compliance. 
The tail shows a smooth swinging characteristics comparable to the one gained by simulation. This behavior results from the compliance of the surrounding water (extrinsic compliance) in combination with a tuneable compliance in terms of the adjustable spring connection between each module (intrinsic compliance); in addition with the inertia of the modules. Thereby the extrinsic compliance disturbs the swinging tail: The disturbance has to be encountered by the intrinsic compliance and the inertia.

Therewith the swinging characteristics can be influenced by either changing the inertia of the segments or altering the spring stiffness of specific joinings. Both alternatives are feasible with the introduced modular robot.

To evaluate the results of the executed simulation, the effector modules have to be tested in combination with the basic structure. Therewith the validation concerning the initial established assumptions of the robot is possible. In addition power consumption and swimming velocity tests need to be done.

By following the idea of a versatilely deployable swimming robot, additional effector modules are required. Taking inspiration from the remaining classes of steady swimming fishes, these modules allow the investigation of carangiform, thunniform and ostraciiform swimming modes. Differences and similarities can be figured out and analyzed.

\section{Conclusion}

This paper introduces a single actuated modular swimming robot. It is able to perform different swimming modes according to steady swimming fishes. The robot consists of two different kinds of modules: The basic structure creates the required torque and determines the direction. The effector modules enable the specific swimming mode and generate the propulsive forces.

By now, the design of the effector modules features a slow anguilliform swimming style. Based on a biological research a mathematical model is introduced. Therewith important data for the design process are evaluated, like required torque and spring stiffness of each joint. The latest design tends to a tail with three modules. The contrast of the practical tests and the simulation shows a well fitting similarity in the swinging behavior.

\section{Acknowledgements}

We gratefully acknowledge the technical support of Danja Voges, Eberhard Hamatschek and Michael Bastick.

\section{References}

[1] Brüggen, N. (1992). Technik der U-Boot Modelle. Verlag für Technik und Handwerk, Baden-Baden.

[2] Crespi, A., Badertscher, A., Guinard, A. \& Ijspeert, A. (2005). AmphiBot 1: an undulant snake-like robot. Robotics and Autonomous Systems 50, pp. $163-175$. 
[3] Fremerey, M., Fischheiter, L., Mämpel, J. \& Witte, H. (2009). Design of a single actuated, undulant swimming robot. The 3rd International Symposium on Mobiligence, (pp. 174-178). Awaji, Japan.

[4] Graham, J. B. \& Lowell, W. R. (1986). Surface and subsurface swimming of the sea snake pelamis platurus. Scripps Institution of Oceanography, University of California, San Diego, USA: Physiological Research Laboratory and Marine Biology Research Division.

[5] Hering, E. (2007). Physik für Ingenieure. Springer Verlag.

[6] VDI (2004). VDI 2206. Düsseldorf.

[7] Laulder, G. V., \& Tytell, E. D. (2005). Hydrodynamics of undulatory propulsion. Fish Biomechanics Vol. 23.

[8] Mämpel, J., Eisold, R., Kempf, W., Schilling, C. \& Witte, H. (2009). A Modular Concept for a Biologically Inspired Robot. Robot Motion and Control, pp. 391-400.

[9] Mämpel, J., Koch, T., Koehring, S., Obermaier, A. \& Witte, H. (2009). Concept of a modular climbing robot. IEEE Symposium on Industrial Electronics and Applications (ISIEA), Kuala Lumpur, Malaysia, pp. 1-6.

[10] Sfakiotakis, M., Lane, D. M. \& Davies, J. B. (1999). Review of fish swimming modes for aquatic locomotion. IEEE JOURNAL OF OCEANIC ENGINEERING, vol. 24 , pp. 237-252.

[11] Zhang, H. X., Gonzalez-Gomez, J., Chen, S. Y., Wang, W., Liu, R., Li, D. et al. (2007), A Novel Modular Climbing Catapillar Using Low-frequency Vibrating Passive Suckers, Proceeding of AIM

[12] Hirose Fukushima Robotics Lab, Japan, Available: http://wwwrobot.mes.titech.ac.jp/robot/snake/acm-r5/acm-r5_e.htmlatm.com

[13] DFKI, Bremen, Germany, Available: http://robotik.dfki-bremen.de/de/ forschung/projekte/unterwasserrobotik/schlange.html 